CORRIGENDUM

Corrigendum: An educational path for the magnetic vector potential and its physical implications

To cite this article: S Barbieri et al 2014 Eur. J. Phys. 35

View the article online for updates and enhancements.
Related content

- $\frac{\text { Reply to Comment on 'An educational path }}{\text { for the magnetic vector potential and its }}$
$\frac{\text { physical implications' }}{\text { S Barbieri, M Cavinato and M Giliberti }}$
- An educational path for the magnetic
$\frac{\text { vector potential and its physical }}{\text { implications }}$
S Barbieri, M Cavinato and M Giliberti
- Comment on 'An educational path for the
magnetic vector potential and its physical
implications'
José A Heras




\title{
Corrigendum: An educational path for the magnetic vector potential and its physical implications
}

2013 Eur. J. Phys. 341209

\author{
S Barbieri ${ }^{1}, M_{\text {Cavinato }}{ }^{2}$ and $M$ Giliberti $^{2}$ \\ ${ }^{1}$ Dipartimento di Fisica e Techologie Relative, Università degli Studi di Palermo, \\ Palermo, Italy \\ ${ }^{2}$ Dipartimento di Fisica, Università degli Studi di Milano, Milano, Italy \\ E-mail: marco.giliberti@unimi.it
}

Received 25 November 2013

Accepted for publication 25 November 2013

Published 17 January 2014

Equation (3) of Barbieri et al (2013 Eur. J. Phys. 34 1209) is incorrect since it has been written in terms of the retarded time $t^{\prime}$ instead of the present time $t$.

Therefore, in place of the following:

$$
' \mathbf{B}(\mathbf{r}, t)=\frac{\mu_{0}}{4 \pi} \int_{V^{\prime}} \frac{\left[\mathbf{J}\left(\mathbf{r}^{\prime}, t^{\prime}\right)+\varepsilon_{0} \frac{\partial \mathbf{E}\left(\mathbf{r}^{\prime}, t^{\prime}\right)}{\partial t}\right] \times \Delta \mathbf{r}}{(\Delta r)^{3}} \mathrm{~d} V^{\prime},
$$

where $V^{\prime}$ is the region containing the currents and

$$
\Delta \mathbf{r} \equiv \mathbf{r}-\mathbf{r}^{\prime}, \quad \Delta r \equiv|\Delta \mathbf{r}|, \quad t^{\prime} \equiv t-\frac{\Delta r}{c},
$$

where $t^{\prime}$ is the retarded time. If we now adopt the quasi-static approximation, that is if we consider only fields that are slowly varying in time, we can neglect all the time derivative multiplied by $1 / c$ (but not time-dependent terms alone). Therefore the contribution of the displacement currents in equation (3) can be disregarded, thanks to the presence of the constant $\varepsilon_{0} \mu_{0}=1 / c^{2}$ that multiplies the time derivative of $\mathbf{E}$. Moreover, the retarded time $t^{\prime}$ of equation (4) also can be considered equal to $t$.',

please read:

$$
\text { 'B }(\mathbf{r}, t)=\frac{\mu_{0}}{4 \pi} \int_{V^{\prime}} \frac{\left[\mathbf{J}\left(\mathbf{r}^{\prime}, t\right)+\varepsilon_{0} \frac{\partial \mathbf{E}\left(\mathbf{r}^{\prime}, t\right)}{\partial t}\right] \times \Delta \mathbf{r}}{(\Delta r)^{3}} \mathrm{~d} V^{\prime},
$$

where $V^{\prime}$ is the region containing the currents and

$$
\Delta \mathbf{r} \equiv \mathbf{r}-\mathbf{r}^{\prime}, \quad \Delta r \equiv|\Delta \mathbf{r}|
$$


If we now adopt the quasi-static approximation, that is if we consider only fields that are slowly varying in time, we can neglect all the time derivatives multiplied by $\varepsilon_{0} \mu_{0}=1 / c^{2}$ (but not time-dependent terms alone). The contribution of the displacement currents in equation (3) can, therefore, be disregarded.'

The mistake in equation (3) of Barbieri et al (2013 Eur. J. Phys. 34 1209) does not influence any of the results or conclusions of the original paper. 